\title{
Study on the relation between plastic strain rate and micro movement of dislocation in $\gamma$-TiAl alloy
}

\author{
Weiping Zhao, a , Guotao Zhang ${ }^{2, b}$, Rui Wang ${ }^{3}$ and Xiaohui Wang ${ }^{\mathrm{c}}$ \\ ${ }^{1}$ Lanzhou Exeter Electro-mechanical Science \& Technology Co. Ltd, Lanzhou, 730050, China \\ ${ }^{2}$ School of Mechanical and Electronical Engineering, Lanzhou University of Technology, Lanzhou, \\ 730050, China \\ ${ }^{3}$ Jiuquan Road Subdistrict Office of Lanzhou, Lanzhou, 730030, China \\ a379302870@qq.com, bzgtly0723@163.com(Corresponding author), \\ cwangxiaohui2666@163.com
}

Keywords: $\gamma$-TiAl alloy; dislocation; slip velocity; slip system; strain rate

\begin{abstract}
The macro-deformation of crystal attributes the micro-slip in crystal of $\gamma$-TiAl alloy. So it is important to reveal the relation between macro-deformation and micro-slip of dislocation. In order to illustrate this relationship, the geometry relation between macro-deformation and dislocation density/external shear stress was established, which was based on the uniaxial tension model. According to the analysis about the relationship, the expression between strain rate of macro-deformation and dislocation density/external shear stress has been obtained. Besides, the curve between strain rate of macro-deformation and external shear stress has been got.
\end{abstract}

\section{Introduction}

Due to the excellent performance of $\gamma$-TiAl alloy, it has been used widely in aerospace and automobile industries. Those performances of $\gamma$-TiAl alloy that have been widely accepted are as follows[1-3]: low density, high stiffness, high resistance to burning and high strength at high temperatures. Generally speaking, there are many crystal defects existing in $\gamma$-TiAl alloy, such as self-interstitials, vacancies and dislocation, etc. Dislocation is the linear defect and it is one of the most important crystal defects in $\gamma$-TiAl alloy. Dislocations in crystal can be moved by the force produced by the interaction of dislocation, etc. And the velocity of dislocation in crystal can be affected by external force, the purity of crystal, the temperature and the type of dislocation.[4-5] Furthermore, the accumulating of slip dislocation in crystal will lead to the plastic deformation for $\gamma$-TiAl alloy. So it is necessary to reveal the relationship between the slip velocity of dislocation and the plastic deformation for $\gamma$-TiAl alloy.

\section{Relationship between slip velocity of dislocation and plastic deformation}

Relationship between dislocation and plastic deformation. In order to study the relationship between dislocation and plastic deformation for $\gamma$-TiAl alloy, the model of the dislocation forced by external force in cylinder should be established firstly, and it's shown in Fig.1.

In Fig.1, the direction for tension along $P P^{\prime}, \boldsymbol{t}$ is the direction of dislocation slip, $N$ is the normal direction of dislocation slip, $l$ and $l$ ' are the distance between the dislocation and crystal surface before/after the dislocation slip, correspondingly. According to the geometry relationship shown in Fig. 1 , the relation $\mathrm{d} l=\mathrm{d} s \cos \lambda$ can be achieved, and the tension strain of $\gamma$-TiAl alloy can be expressed as follows:

$$
\mathrm{d} \varepsilon=\frac{\mathrm{d} l}{l}=\frac{\mathrm{d} s \cos \lambda}{l}
$$

The shear strain caused by the slip of dislocation is: 


$$
\mathrm{d} \gamma=\frac{\mathrm{d} s}{l \cos \varphi}
$$

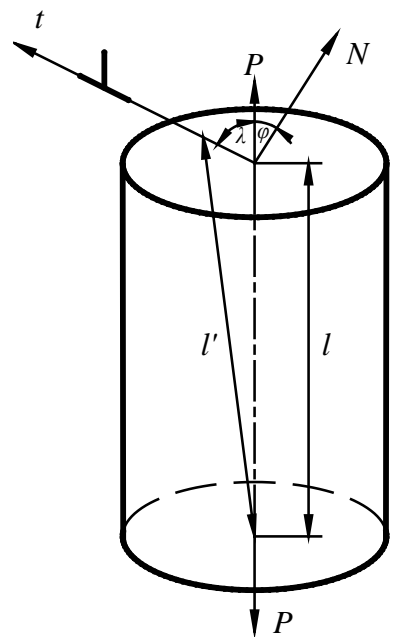

Fig.1 Model of dislocation slip in crystal

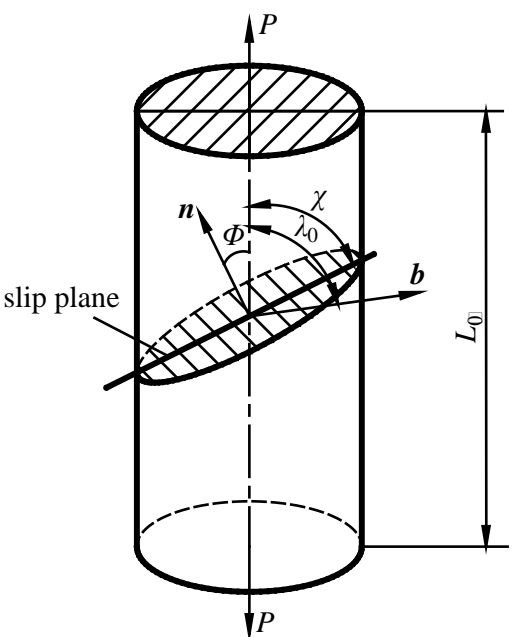

Fig.2 Model of uniaxial tension

So the strain rate caused by dislocation slip can be accepted, and it can be expressed as follows:

$$
\mathrm{d} \varepsilon=\cos \varphi \cos \lambda \cdot \mathrm{d} \gamma=\Omega \cdot \mathrm{d} \gamma
$$

where the $\Omega=\cos \varphi \cos \lambda$ is the Schmid factor, $\varepsilon$ is the strain caused by dislocation slip in the crystal of $\gamma$-TiAl alloy.

Let the density of dislocation in $\gamma$-TiAl alloy is $\rho_{d}$, and the displacement of dislocation slip is $\mathrm{d} x$, so a new expression is achieved:

$$
\mathrm{d} \gamma=\rho_{\mathrm{d}} b \mathrm{~d} x
$$

Substituting from the expression (4) into the formula (3) gives:

$$
\mathrm{d} \varepsilon=\rho_{\mathrm{d}} b \Omega \mathrm{d} x
$$

So

$$
\dot{\varepsilon}=\frac{\mathrm{d} \varepsilon}{\mathrm{d} t}=\rho_{\mathrm{d}} \Omega \frac{\mathrm{d} x}{\mathrm{~d} t}=\Omega \rho_{\mathrm{d}} b \bar{v}
$$

where $\bar{v}$ is the average speed of dislocation glide in $\gamma$-TiAl alloy, $\dot{\varepsilon}$ is the strain rate of plastic deformation of $\gamma$-TiAl alloy.

Relationship between plastic deformation and external force. The relation between average speed and the external force of dislocation glide for $\gamma$-TiAl alloy at room temperature is[4]:

$$
\bar{v}=\frac{b}{B} \tau
$$

where $B$ is the frictional coefficient and the value of $B$ ranges from 0.1 to $1000 \mathrm{~m} \cdot \mathrm{s}^{-1}$.

Substituting from (6) into (5) gives:

$$
\dot{\varepsilon}=\frac{1}{B} \Omega \rho_{\mathrm{d}} b^{2} \tau
$$


Furthermore, the geometry relationship between dislocation density and the force along slip plane for $\gamma$-TiAl alloy is shown as follows:[6]

$$
\tau=\tau_{0}+k \rho_{\mathrm{d}}^{1 / 2}
$$

where $k$ is constant and $\tau_{0}$ is the shear stress of $\gamma$-TiAl alloy with no defects.

(i) Substituting from (8) into (7), gives

$$
\dot{\varepsilon}=\frac{1}{B k^{2}} \Omega b^{2}\left(\tau-\tau_{0}\right)^{2} \tau
$$

Let the shear modulus of $\gamma$-TiAl alloy adopted in this paper is $68.57 \mathrm{GPa}$, and the result that $\tau_{0} \approx 68.57 / 2=34.285 \mathrm{GPa}$, inserting it into the expression (9), and then

$$
\dot{\varepsilon}=\frac{1}{B k^{2}} \Omega b^{2}(\tau-34.285)^{2} \tau
$$

Based on the expression (10), the relation between strain rate of macro-deformation and the external force in $\gamma$-TiAl alloy has been obtained. This relation will provide the theoretical basis for us to study the plastic deformation of $\gamma$-TiAl alloy.

(ii) Substituting from (8) into (7), and another expression can be obtained:

$$
\dot{\varepsilon}=\frac{1}{B} \Omega \rho_{\mathrm{d}} b^{2}\left(\tau_{0}+k \rho_{\mathrm{d}}^{1 / 2}\right)=\frac{1}{B} \Omega \rho_{\mathrm{d}} b^{2}\left(34.285+k \rho_{\mathrm{d}}^{1 / 2}\right)
$$

According to the expression (11), the relation between the strain rate of macro-deformation and dislocation density in $\gamma$-TiAl alloy has been revealed.

Analysis of the relationship between macro-deformation and the external force. Based on the geometry model with unaxial tension for $\gamma$-TiAl alloy shown in Fig.2, the crystal geometry relationship can be obtained, besides, the Schmid factors of the main slip system also can be calculated, and they are listed in Table 1.

Table 1 Schmid factors of the main slip system

\begin{tabular}{ccc}
\hline slip system & (plane)[direction] & $\Omega=\cos \varphi \cos \lambda$ \\
\hline & $(111)[1 \overline{1} 0]$ & $-1 / 3 \sqrt{6}$ \\
main slip system & $(111)[\overline{1} 01]$ & $-2 / 3 \sqrt{6}$ \\
& $(111)[0 \overline{1} 1]$ & $-1 / 3 \sqrt{6}$ \\
\hline
\end{tabular}

Let $B=1 \mathrm{~m} \cdot \mathrm{s}^{-1}$ and $b=0.2888 \mathrm{~nm}$. In order to simplify the analysis, the value $\Omega=-1 / \sqrt{6}$ is adopted as an example to illustrate the relation. Inserting the three parameters into the expression (10), and then the curve between $\dot{\varepsilon}$ and $\tau$ for $\gamma$-TiAl alloy can be got, and it's shown in Fig.3.

According to the curve shown in Fig.3, the macro-deformation rate $\dot{\varepsilon}$ will be increase firstly and then decrease with external force $\tau$ ranges from 0 to the shear modulus. Besides, $\dot{\varepsilon}$ will be zero when the external force $\tau$ equals the shear modulus, which indicates that $\gamma$-TiAl alloy has been fractured. Furthermore, $\dot{\varepsilon}$ will be the maximum when $\tau=2 / 3 \mu=45.7 \mathrm{GPa}$, and then the fatigue life of tension life of the specimen will be shorter than other condition. 


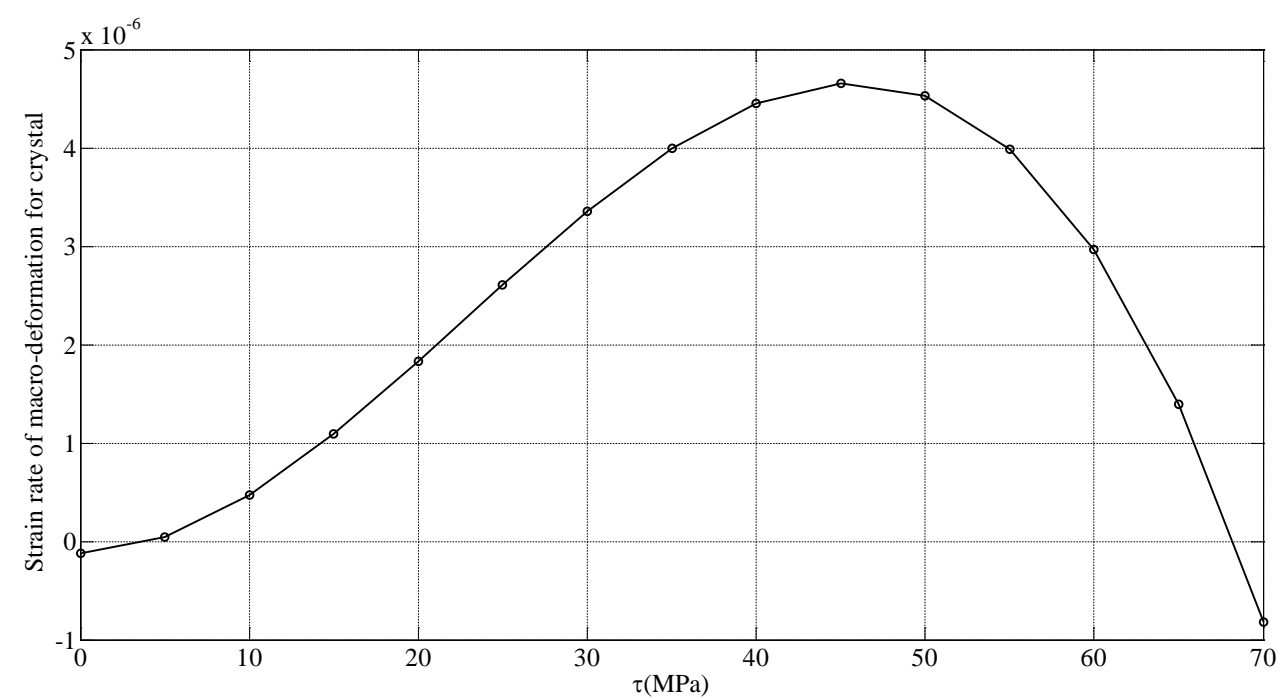

Fig.3 Relation curve between strain rate and external shear stress

\section{Conclusion}

$\gamma$-TiAl alloy is known to be major raw materials for manufacturing the mechanical parts operating at high temperature, and also plays a vital role in modern industries. Plastic deformation is the macrodeformation, and its nature is the micro-movement in $\gamma$-TiAl alloy crystal. Some conclusions based on the analysis can be obtained: (1) The relation between dislocation density and plastic deformation has been revealed; (2) The relation between plastic deformation and external shear stress has been got; (3) The relation curve between macro-deformation rate and external shear stress for $\gamma$-TiAl alloy has been given.

\section{Acknowledgements}

This work was supported by the Program for Changjiang Scholars and Innovative Research Team in University of Ministry of Education of China(IRT1140).

\section{References}

[1] F.C. Feng, Z.Y. Rui, G.T. Zhang, C.F. Yan and X.B. Yi: Strength of Materials. Vol. 46(2014), p. 183.

[2] Zhang Guotao, Rui Zhiyuan, Feng Ruicheng, Zuo Yanrui and Yan Changfeng: Applied Mechanics and Materials. Vols. 547-458(2014), p.19.

[3] Helmut Clemens and Svea Mayer: Advanced Engineering Materials, Vol. 15(2013), p191.

[4] D. Hull and D.J. Bacon: Introduction to dislocation (Fifth Edition, Elsevier, UK 2011).

[5] W.G. Johnstok and J.J. Gilman: Journal of Applied Physics. Vol. 30 (1959), p129.

[6] Reza Abbaschian, Lara Abbaschian and Robert E. Reed-Hill: Physical metallurgy principles (Fourth Edition, Cengage Learning, The United States of America 2008). 